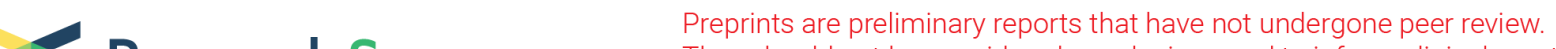 Research Square
They should hot be considideded conllusivive, used to inform clinical practice,
or referenced by the medias validated information.
}

\section{Attitudes of Neurologists to Brain Death and Organ Donation in China: A Content Analysis}

\section{Ping Wang}

Department of Neurology, The Second hospital ,Cheeloo college of medicine,Shandong University

\section{Yuji Guo}

Department of Histology and Embryology,Cheeloo college of Medicine,Shandong University

\section{Gaohui Yin}

School of translation studies $₫$ QuFu normal university

\section{Mengzhen Li}

Qufu Normal University

Yan Li

Department of neurology $₫$ YiShui County People's Hospital

\section{Nan Li}

Department of neurology $\llbracket$ SiShui County Hospital

\section{Suqin Jin}

Department of neurology, the Second Hospital Cheeloo college of Medicine,Shandong University

\section{Zhengyu Zhu}

Department of neurology,cheeloo college of medicine ,Shandong University

\section{Mao Ding}

Department of Neurology,The Second Hospital, Cheeloo college of medicine Shandong University

\section{Bingcong Chu}

Department of Neurology,the second hospital,Cheeloo college of medicine,Shandong University

\section{Yijin Wu}

School of translation studies ,Qufu normal university

\section{Wen Ma ( $\triangle$ mawen@sdu.edu.cn )}

Shandong University, Jinan, China

\section{Research article}

Keywords: Brain death, Organ donation, Content analysis, Traditional culture, Attitudes

Posted Date: December 28th, 2020

DOI: https://doi.org/10.21203/rs.3.rs-124139/v1 
License: (c) (i) This work is licensed under a Creative Commons Attribution 4.0 International License. Read Full License 


\section{Abstract}

Background: Between 2003 and 2009, there were approximately 20,000 brain deaths per million people per year in China.These cases represent an important source for organ donation. As it is neurologists who are primarily involved in determining brain death, their knowledge and attitude toward brain death and organ donation directly affects decisions of family members regarding organ donation.

Methods: This qualitative study was conducted in 2019. Data on neurologists' knowledge and attitudes towards brain death and organ donation were obtained from fifteen neurologists in the Departments of Neurology from Chinese hospitals using a content analysis approach as achieved in semi-structured interviews.

Results: Overall, these neurologists conveyed a positive attitude toward organ donation after brain death. However, they noted the importance of considering and respecting the opinions of these patients' families. From the data analysis, 4 themes and 12 categories were developed. The 4 themes included: 1) brain death organ donation as a good thing, 2) interacting with brain death patient's family, 3) cultural influences on organ donation with brain death and 4) strategies to improve organ donation rates in patients with brain death.

Conclusion: The results of this study have provided some new and important information regarding the knowledge and attitude of Chinese neurologists toward brain death and organ donation. Such information is critical for guiding government and health care systems in designing strategies to improve donation rates and decisions from brain death patients.

\section{Background}

Brain death is an irreversible cessation of function of the entire brain and is considered as the medically and legally accepted verification of death in a number of countries including the United States, Japan, Germany, the United Kingdom and India. [1-3]. Interestingly, in China no legal status regarding brain death currently exist, nor is the concept of brain death widely accepted by Chinese people [4, 5]. Most Chinese consider that death involves the irreversible cessation of circulatory and respiratory function, which is then referred to as non-heart-beating death [6]. Thus, Chinese are less likely to donate organs after brain death, which is one of the most notable reasons for shortages in transplanted organs $[5,6]$. Between 2003 and 2009, there were approximately 20,000 brain deaths per million people per year, however, there were only 61 brain dead donors in China over this period [7]. While hundreds of millions of dollars are expended annually on brain death patients in China, the excessive number of organs potentially available from these patients were not harvested for transplantation.

Although no legislation on brain death currently exists in China, the National Health and Family Planning Commission has released a Criteria for Clinical Determination of Brain Death, which now provides guidelines on how to define brain death (Brain Injury Evaluation Quality Control Centre of National Health and Family Planning Commission 2013). The determination of brain death in China has been made in 
accordance with internationally accepted standards for determining brain death [8]. Some examples of standards which have been incorporated include the suggestion that transplant physicians should not be involved in the determination of brain death, to preclude the possibility that transplant physicians would employ unethical determinations to acquire more transplantable organs. In addition, two neurologists should be involved in determining the brain death [9]. As a result of these recommendations, an increasing number of patients are declared brain dead annually in China, which means that neurologists are working with more brain-dead patients and their family members. Therefore, the knowledge and attitude of neurologists toward brain death and organ donation will directly affect their input into decisions of family members regarding donation of their relatives' organs. However, this issue has received little, if any, attention. In this study, a content analysis method was used as an approach to assess the knowledge and attitude of Chinese neurologists toward brain death and organ donation.

\section{Methods}

\section{Study Design}

Semi-structured face-to-face interviews were conducted with neurologists. A content analysis method was then used to describe and interpret the knowledge and attitude of these Chinese neurologists toward brain death and organ donation.

\section{Study Participants}

Participants in this study included 15 neurologists working in the Department of Neurology of the Second Hospital of Shandong University and two other county hospitals in Shandong province, China. These included 11 women and 4 men, with ages ranging from 27 - 49 and an average age of 37.7 years. Of these 15 neurologists, 11 were from the Second Hospital of Shandong University and included residents, attending physicians and associate chief physicians. Three had more than 20 years experience, 3 more than 10 years, 3 more than 5 years and 2 less than 5 years. Of the 4 neurologists from the county hospitals, 2 had more than 20 years experience and 2 more than 10 years. The education levels of these neurologist consisted of 6 doctoral, 7 postgraduate and 2 undergraduate students(Table 1). All participants were fluent in speaking and writing Chinese and were able to complete the semi-structured interview within 25-40 minutes.

\section{Data Collection}

All neurologists agreed to participate in this study and submitted formal consents. Interviews were conducted at the Second Hospital of Shandong University and the Yishui and Sishui County Hospitals of Shandong province over the period from October to December, 2019. Names of the participants were not disclosed, but other characteristics were collected as summarized in Table 1. The purpose of the study was described to each participant prior to the interview. Based on Chinese national conditions and 
culture, we designed the questionnaire and interview guide (table 2). Each participant was interviewed individually in an one-on-one format and consisted of open-ended questions . Participants were encouraged to talk about their knowledge and attitude toward brain death and organ donation. All interviews were audio-recorded and typically lasted $25-40$ minutes. The recordings were then transcribed into written forms and repeatedly analyzed until specific themes were identified.

\section{Data analysis}

In this study, an inductive content analysis method was used to analyze and interpret the interview data[10,11,12] . The analytical procedures consisted of five parts: 1) all transcribed data were reviewed independently by all researchers to ensure accuracy of the data transcription, 2) after identifying the unit of analysis such as words, phrases, and sentences, researchers determined which quotations should be included or excluded in the research results, 3 ) the lead investigator summarized the codes concerning the knowledge and attitudes of neurologists toward brain death and organ donation, 4) input from all researchers was coalesced to establish and confirm the validity of the identified themes and sub-themes and 5) all themes and sub-themes were described and analyzed in detail.

\section{Results}

Four themes and twelve sub-themes related to Chinese neurologist's knowledge and attitudes toward brain death and organ donation were identified as a result of this study (Table 3). Each are discussed in detail in the following sections.

\section{Theme 1: Brain death organ donation as a good thing}

All participants expressed positive support for brain death organ donation. Three of the most important factors included benefits to recipients, benefits to donors and benefits to medical institutions.

\section{Benefits to recipients}

Donating organs after brain death serves as an effective means for saving the lives of recipients.

Saving their lives. One of the most acknowledged benefits of brain death organ donation was that of saving and/or improving the quality of the recipient's life. Medical professionals regard organ donation after brain death as a noble deed that should be respected as it provides hope for survival in many families on the brink of despair.

It's a pretty good action to donate organs after brain death because it could provide organs for patients who need an organ transplant to keep alive. -P4 
It may be the greatest opportunity for patients on the transplantation waiting list to obtain a new life. $-\mathrm{P} 9$

Obtaining well-functioning organs. Along with the increasing normalization of criteria for judging brain death, medical specialists unanimously accept that brain death is death in the true sense. However, organs from these brain dead patients must be in an well condition of function before being considered for transplantation.

The organs of individuals with brain death is the optimal one for transplanting. $-\mathrm{P} 8$

I am very supportive of organ donation after brain death. I believe that many organs of patients with brain death still function well. Donating organs after brain death is a good deed for other patients who are argent for organ transplantation. -P10

I advocate brain-dead patients donating organs over certain reasons that their organs are optimal transplant ones. It could bring survival opportunities to others. $-\mathrm{P} 11$

\section{Benefits to donors}

Brain death is irreversible. Thus, donating organs after diagnosis of brain death provides an opportunity for an extension of the recipient's life. Simultaneously, this noble gesture would improve the value and purpose of the donor's life.

Improving life's value. The Chinese regard helping others as one of the norms of social moral behavior. Accordingly, a person who utilizes their best efforts to aid others would be regarded as a model citizen. Such a person would then achieve an increased appreciation for the value of life and be respected in Chinese society.

Organ donation can indeed save a life or even a family. The life value of patients with brain death would be improved and their lives would be prolonged. $-\mathrm{P} 6$

I agree a lot about organ donation of brain death patients. We should consider not only the length of our lives but also life quality. Donating organs help donors achieve their life value . -P12

Life extension. As organs from brain dead individuals function in the recipient, in effect, a portion of the donor's life continues on.

I believe donating organs after brain death is an action to continue life. $-\mathrm{P} 5,8,9,11$

\section{Benefits to medical institutions}

Participants noted that prolonging the treatment of brain dead patients for a few days was ineffective and only consumed medical resources which could better serve others. In contrast, organ donation 
ensures a more reasonable allocation of medical resources and offers opportunities for the delivery of functional transplants, thus facilitating medical and societal progress.

Organ transplantation after brain death may make a greater contribution to the entire society by promoting the progress of medical science. Besides, brain death organ donation could save medical resources, thus helping many other patients. $-\mathrm{P} 10$

\section{Theme 2 Interacting with family members of brain dead patients}

The participants agreed that opinions and inputs of family members must remain a first consideration, as the decision regarding treatment cessation and organ donation is ultimately the charge of family members. The sub-themes that emerged from these data included: 1) no mention of organ donation, 2) suggesting organ donation, 3 ) no mention of terminating treatment and 4) suggesting termination of treatment.

\section{No mention of organ donation}

Most participants indicated that they were reluctant to initiate interactions with family members of braindead patients regarding organ donation for the following three reasons: 1) it may trigger a doctor-patient conflict, 2) doctors lack of knowledge/information regarding organ donation and 3) quality of donor organs.

Triggering doctor-patient conflict. Grieving family members of brain dead patients strongly contravened doctors' suggestions of organ donation.

More often, as doctors in charge, we argued that it is very inappropriate and easy to cause doctor-patient conflict to persuade patients' family members to give up treatment and propound them to donate organs at the same time. $-\mathrm{P} 4,10$

I will not induce family members of brain death to donate organs. It could increase the risk of conflict between doctors and patients. $-\mathrm{P} 4$

Generally speaking, we don't talk about organ donation with family members of brain death since they would disgust these suggestions. -P13

Lack of knowledge regarding organ donation. Several participants reported that they avoided mentioning organ donation when interacting with family members as they felt that they lacked sufficient knowledge and information on the topic.

We lack knowledge about brain death, and we can' $t$ immediately identify the criteria for brain death. -P7 
I have tried countless times to suggest family members of young and middle-aged patients with brain death donate organs but I didn't implement it eventually. One is because they are in grief, and the other is because I do not know the way to donate organs. -P12

Quality of donor organs. The quality of organs available should be considered before initiating a discussion of organ donation with family members.

I rarely recommend organ donation because most of the patients in our department suffer from severe stroke and encephalopathy, and the patients are generally old, the condition and function of organs are not very good. $-\mathrm{P} 7$

\section{Suggesting organ donation}

Depending on the extent and details of communication that exists between doctors and the brain dead patients' family members, some doctors will offer the option of organ donation, while considering the families' input and opinions.

I would like to advise families to donate patients' organs but their decisions come first. $-\mathrm{P} 11,14,15$

\section{No mention of terminating treatment}

Participants rarely suggest that treatments should be terminated until it is obvious to family members that treatments are no longer effective and serve no purpose.

When I found out that the family took the initiative to give up treatment, I would tell them carefully about the prognosis of brain death patients. But I will never take the initiative to propose that the treatment is meaningless. $-\mathrm{P} 4$

It's better to follow the wishes of the families of patients so I rarely persuaded patients to give up treatment. $-\mathrm{P} 7$

\section{Suggesting termination of treatment}

Participants sometimes suggest that families terminate treatment as the brain death experienced by the patient is irreparable. Therefore, continuing treatment of such patients unnecessarily consumes considerable manpower and medical resources which is not conducive to the welfare of society and citizens.

Increasing unnecessary financial burden on families. As prolonged treatment of brain dead patients is ineffective and costly, it exerts undue financial burdens upon these families. 
Continued treatment of patients with brain death will bring a heavy burden on the family and society. So, I generally recommended family members to give up treatment. $-P 5,10$

Consuming medical resources. The absence of any potential benefits of medical treatments to support brain dead patients results in an unreasonable allocation of medical resources.

For society, the excessive consumption of such medical resources in the treatment of brain-dead patients is not very reasonable for the fairness of the entire medical resources. $-P 5,10$

\section{Theme 3 Cultural influences on organ donation with brain death}

Traditional culture plays an irreplaceable part in making decisions regarding organ donation. This theme explores the positive and negative influences of traditional Chinese culture on organ donation from brain dead patients.

\section{Incentives from traditional cultural influences}

As a result of the long and profound history of Chinese culture there exist a number of factors that drive decisions to donate.

From our discussions with all participants, it seems that the values and beliefs they perceived as coming from the family members contributed significantly to these family members' decisions about organ donation. Traditional values can be imbued with positive messages. For example, a spirit of selfless dedication, mercy, as well as a sense of fellowship, all exert an overpowering impact on daily life and act as an impetus for organ donation after brain death [13].

I think there exist good motivators for organ donation with brain death in Chinese traditional culture. For instance, we have been promoting the spirit of selfless service and doing more for others. This kind of values is also an essential part of traditional culture, so I think that we need to keep what is good. P6

\section{Disincentives from traditional culture influences}

In addition to positive effects, many participants shared their experiences with traditional ideas relating to refusals for organ donation. Some responses in this regard included a diversity of thinking in the areas of body integrity, completist and filial piety.

\section{Body integrity}


There is a popular saying in China about keeping the body intact: "When a person is buried in the soil after death, the dead will rest in peace, and the living will feel at ease." Based on this traditional concept of burial, the Chinese are more prone to maintain an intact body. From the discussions with the participants, body integrity was considered as a major factor contributing to an unwillingness to donate.

For the older generations, they know little about organ donation, and some traditional concepts are firmly rooted in their minds. For example, we must keep our bodies complete and bury them after death. -P4

The transplant violates the traditional view that we can't hurt our bodies. -P5

Sometimes Chinese would like to burn paper money to commemorate the ancestors and show love to them. They think that they live in another world, so if they die with incomplete bodies, they will be the same there. $-\mathrm{P} 6$

\section{Family ties}

Many participants expressed that interactions among family members represented a notable factor regarding their final decision. In spite of their initial opinions regarding organ donation, involvement of inputs from all family members played an important role in the process of making a decision.

Many people are very scrupulous when they consider whether they can donate the remains, and worry that these things they do will be criticized by relatives and friends. In fact, this kind of pressure is very great for family members, and they may be blamed for not being filial enough, and not caring for their children. Some people even believe that they are doing these things for some financial benefits. -P4

As a direct relative of the patient, if the child has passed away, it may be better for the parents to make a decision, and the blame may be less. But if the parents die and the children make decisions, the pressure will be great. Sometimes, When a husband dies, it is not just the wife who makes the decision. The opinions of other family members often influence the final decision and interfere with organ donation.. --P7

\section{Filial piety}

Confucius had emphasized that we are from our parents. Therefore, out of filial piety for our parents, we are not to damage our body, even hair and skin. Without following this philosophy, Chinese experience a feeling that they are unfilial.

Filial piety is the most important of all virtues, so if we donate the organ after our parents die, we'll be considered unfilial. -P10

The basic requirement in filial piety culture is to keep bodies integral. -P5 


\section{Theme 4 Strategies to improve organ donation rates in brain dead patients}

Given these factors that affect the reality of organ donation in China, it remains clear that increasing the number of organ donations would narrow the gap between the demand and donated organs [14]. This theme encompasses the government, the people involved and the medical institutions which can all contribute to various aspects on the improvement in organ donation rates.

\section{Government}

It is critical that the government take a leadership role on this issue. A large number of participants shared their impressions that many family members do not have a complete understanding of organ donation and the laws regarding organ donation with brain dead patients. Based on discussions with the participants, four strategies emerged which might lead to an increase in organ donation.

\section{Strengthening the education}

In China, discussions concerning death seldom occur in conversations among family members, which results in a lack of education on the topic of death[15]. From the interviews, many participants mentioned that most of the family members of brain dead patients lacked a thorough understanding of the concept of brain death and organ donation. Consequently, many relatives expressed a reluctance to donate. It was felt that there is a an urgent need to strengthen the education on this issue.

Strengthening education and increasing knowledge of it can play a certain role in improving some traditional concepts. --P8

\section{Amending related laws and regulations}

Current laws and related regulations for organ donation are sorely inadequate. For example, no laws exist for establishing a clear criteria for the diagnosis of brain death. This becomes a crucial element contributing to the number of organs donated from brain death patients.

The organ donation after brain death should be regulated in laws or policies and regulations to dispel public doubts, such as the identification of brain death. There are many methods for brain death identification, but I think the brain death identification agency should be established from a national perspective. --P4

First of all, the location of organ donation needs to be regulated, and the standard for brain death needs to be legislated. --P5 


\section{Providing reasonable incentives}

Overall, there remains a negative attitude toward organ donation after brain death. It seems likely that the addition of reasonable incentives, for example, making the process more honorable and reducing medical expenses for families with financial problems, would increase the appeal of donation.

From the government's point of view, I think we should add a reward to let everyone realize that it is a very glorious thing. -P4

I think the government should provide some financial support to people who donate organs. In clinical practice, most of our patients need to spend a lot of money in the course of treatment, except for emergencies such as trauma. I think if the government can provide financial support, it can directly increase the organ donation rate. $-\mathrm{P} 6$

\section{The masses}

The power of the mass media should not be underestimated. In this way, measures directed at promoting organ donation within the Chinese population may enable an increase in rates of donation..

The social media should play important role to popularize the knowledge, and let people know it's extremely meaningful. --P4

From the perspective of society, more people are needed to publicize this kind of knowledge of organ donation. Let the concept of organ transplant take root in the hearts of the people. -P5

\section{Health care system}

Another major component is that associated with the health care system, that can play a differential and crucial role.

Improving medical procedures. For effective organ donation, in addition to location of required organs, a critical factor is the medical techniques available. In this regard, it is the responsibility of medical institutions to improve their medical procedures and standards as a means to increase survival rates of organ transplants.

In my opinion, we must first increase the success rate of organ transplants. -P6

Providing humanistic care. In the decision making process involved with organ donation there is a substantial degree of pressure and psychological trauma experienced by the family and related individuals. Therefore, while the medical staff can alleviate the anguish of the disease, this should be accompanied with some counseling.

Medical staff provide psychological counseling to family members of brain-dead patients. -P4 
Pay attention to humanistic care, and do a good job of organ donors and their families before, during and after organ donation. -P11

\section{Discussion}

Overall, the neurologists recruited for this study expressed a positive attitude toward organ donation after brain death. They regard organ donation after brain death as a noble gesture which was beneficial for recipients, donors and the medical institution. For recipients, the obvious advantage is a supply of wellfunctioning organs that saves and/or improves the quality of their lives. For donors, their value in society is enhanced and a portion of their life extended owing to the functioning of their organs within recipients. For the medical institution, the transplant procedure serves as an effective utilization of medical resources and contributes to medical progress.

Participants in our study generally agreed that doctors should respect the opinions of the patients' families. When interacting with patients' family members, they usually avoid initiating discussions about organ donation and cessation of treatment. Three major reasons given for evading mention of organ donation to the patient's family included the possibility that this will trigger a doctor-patient conflict, the doctors lack of knowledge on organ donation and concerns regarding the potential quality of the organs. Under conditions where an extended and detailed interaction exists between the doctor and family members, some doctors are more likely to suggest the termination of treatment along with the donation of organs. In these cases, the doctor explains the irreversible nature of the brain death and that treating brain dead patients unnecessarily consumes medical resources and adds to the burden exerted on the families.

The traditional culture that exist in China significantly influences beliefs as related to brain death and organ donation[16]. Most of the participants note that these traditional cultural beliefs can act as a barrier to this topic. However, there are many idioms and allusions in the traditional culture that promote the attitude to save a life. For example, the giving of oneself to save others, the righteousness nature of this act and the idea of cutting off stocks to serve the emperor are all in accord with the ancestral view of life and death and the spirit to save people.

In this study, strategies to improve the donation rate from brain dead patients are presented. Participants proposed some sources including the government, mass media exposure and improvements in health care procedures which could be incorporated to increase organ donation rates. Almost all participants mentioned that approaches to raise awareness and enhance knowledge about this topic would serve to popularize and thus increase organ donations. Besides, it's crucial to provide institutional guarantee for both donors and receipts. Moreover, standardizing the whole process would also greatly aid in the promotion of organ donations from brain dead patients. 


\section{Conclusion}

In this study we present the attitudes of Chinese neurologists toward organ donation from brain dead patients. The responses of these neurologists provide some novel and critical information to guide government and health care system institutions in the development of strategies to improve donation rates from brain death patients.

\section{Declarations}

\section{Ethics approval and consent to participate}

This study was approved by the Ethics Committee of the second hospital of Shandong University (Approval number:KYu-2019(Iw)035). The informed consent form was signed by subjects after informing them of the study objectives, risks and benefits, the voluntary nature of participation in the study and recording of their voices.

\section{Consent for publication}

Not applicable.

\section{Competing interests}

The authors declare that they have no competing interests.

\section{Funding}

Shandong University humanities and Social Sciences major project (Grant No. 19RWZD14) supported the design of the study and collection of data. Shandong University under grant number sy20202401 and Shandong Province key Research and development project (Grant No.2019GSF108250) supported the analysis and interpretation of data, and supported write the manuscript .

\section{Authors' Contributions}

$P W, Y G, W M$, and $Y W$ contributed to the conception and study design; $P W$ and $Y G$ performed the experiments. GY and ML performed the content analysis. YL,NL,SJ,ZZ,MD and BC contributed to data collection, data analysis; $P W, Y G, W M$ and $Y W$ wrote the manuscript.

PW and YG contributed equally to this work and should be considered co-first authors.

Correspondence author: Yijin Wu $\otimes$ Wen Ma. 


\section{Acknowledgments}

We thank the neurologists of Second Hospital of Shandong University, Yishui County Hospital and Sishui County Hospital for this interview.

\section{Availability of data and materials}

All datasets generated for this study are included in the article.

\section{Author details}

${ }^{1}$ Department of Neurology, The Second Hospital, Cheeloo College of Medicine, Shandong University , Jinan, China

${ }^{2}$ Department of Histology and Embryology, Cheeloo College of Medicine, Shandong University, Jinan, China.

${ }^{3}$ School of translation studies, Qufu Normal University, Qufu, China

${ }^{4}$ Department of Neurology, YiShui County People's Hospital, Yishui, China

${ }^{5}$ Department of Neurology, People's Hospital of Sishui County, Sishui, China

${ }^{6}$ School of Foreign Languages \& Literature, Shandong University, Jinan, China

\section{References}

1. Smith M. Brain death: The United Kingdom perspective. Semin Neurol 2015;35:145-51.

2. Greer DM, Wang HH, Robinson JD, Varelas PN, Henderson GV, Wijdicks EF. Variability of Brain Death Policies in the United States. JAMA Neurol. 2016;73(2):213-8.

3. Aramesh K, Arima H, Gardiner D, Shah SK. An International Legal Review of the Relationship between Brain Death and Organ Transplantation. J Clin Ethics. 2018;29(1):31-42.

4. Huang J, Wang H, Fan ST, Zhao B, Zhang Z, Hao L, Huo F, Liu Y. The national program for deceased organ donation in China. 2013;96(1):5-9.

5. Yang Q, Miller G. East-West differences in perception of brain death. Review of history, current understandings, and directions for future research. J Bioeth Inq. 2015;12(2):211-25.

6. Liu Z, Zhu B, Yun P, Wang P, Wang X, Xu H. Are we ready to utilize non-heart-beating donors for clinical allotransplantation in China? Transplant Proc. 2008;40(4):1018-20. 
7. Zhou JS., and Dou KF. Confusions and hopes for organ transplant in China. Organ Transplantation 2011,2(3): 241-243.

8. Halevy A, Brody B. Brain death: reconciling definitions, criteria, and tests. Ann Intern Med. 1993,119(6):519-525.

9. Su Y, Yang Q, Liu G, Zhang Y, Ye H, Gao D, Zhang Y, Chen W. Diagnosis of brain death: confirmatory tests after clinical test. Chin Med J (Engl). 2014;127(7):1272-7.

10. Molzahn A, Starzomski R, McDonald M, O'Loughlin Chinese Canadian beliefs toward organ donation. Qual Health Res. 2005;15:82-98.

11. Virginia B, Victoria C. Successful Qualitative Research: A practical guide for beginners. London: Sage Publications $\rrbracket$

12. Webber K, Davies AN, Cowie MR. Breakthrough pain: a qualitative study involving patients with advanced cancer. Support Care Cancer. 2011;19:204-2046.

13. Wu Y, Elliott R, Li L, Yang T, Bai Y, Ma W. Cadaveric organ donation in China: a crossroads for ethics and sociocultural factors. Medicine (Baltimore) 2018;97:e9951.

14. Zhang Z, Yuan X, Wu Y, Guo F, Tian J. The family attitudes of patients with end-stage renal disease toward living kidney donation in China. Transplantation Proceedings,2020,52, 1360-1364.

15. Yang T, Wu Y, Ethical disputes over living organ transplant and the applicable solutions to these disputes in China. Acta Bioethica, 2018; 24ख2):219-225.

16. Wu Y, Yang T, Li L, Ma W. Ethical issues relating to living organ donation in China. Transplantation Proceedings 2016, 48: 1875-1878.

\section{Tables}

Due to technical limitations, tables are only available as a download in the Supplemental Files section.

\section{Supplementary Files}

This is a list of supplementary files associated with this preprint. Click to download.

- Table1.docx

- Table2.docx

- Table3.docx

- supplementary1.docx 